\title{
Model-based Trajectory Control of Robots with Pneumatic Actuator Dynamics
}

\author{
Ryuma Niiyama
}

\begin{abstract}
Pneumatic actuators have many attributes such as natural compliance and high peak power capabilities that make them attractive for research in dynamic legged locomotion. However, the effects of nonlinear flow through the pneumatic components limit the bandwidth of actuators, thus restricting their use in a high-performance control system. We believe that a model-based control design can overcome these bandwidth limitations. In this study, we demonstrate that black-box system identification of actuator dynamics can be effectively combined with nonlinear trajectory optimization and stabilization to accomplish dynamic tasks on underactuated robots. We present two case studies: an underactuated cart-pole system with the cart driven by a pneumatic actuator and a compass gait walking robot with pneumatic toes.
\end{abstract}

\section{INTRODUCTION}

Dynamic legged locomotion requires actuators that are lightweight, high-power, and able to withstand impact forces. Fluid actuators outperform electric actuators in this regard, and have been used in legged locomotion research and applications; for example, hydraulic actuators are used in the rough-terrain quadruped robot[1] and force-controlled humanoid robot[2][3]. Pneumatic actuators, especially pneumatic muscles, are used in the bio-inspired humanoid robot[4], walking biped robot[5], passive-based walking robot[6], and running bipedal robot[7].

Pneumatic actuators have some advantages over hydraulic actuators, such as clean operation, easy handling, natural compliance, and the ability to carry power onboard in compressed air tanks. A number of studies have reported on the modeling and control of pneumatic systems to overcome nonlinear properties resulting from the compressibility of the air and effects of friction. Detailed nonlinear mathematical models have been proposed[8], [9][10], [11]. The primary disadvantage of pneumatic actuators is their low bandwidth when compared to hydraulic actuators. However, humans can perform highly dynamic athletic movements despite being equipped with actuators (human muscles) that have bandwidth limitations similar to those of pneumatic actuators[12]. This fact suggests that low bandwidth does not necessarily imply poor performance; it implies that an actuator cannot be treated as a perfect torque source and the dynamics of the actuator should be considered in motion planning solutions.

In this paper, we propose a control method with modelbased trajectory planning that consider the actuator dynam-

Computer Science and Artificial Intelligence Lab, Massachusetts Institute of Technology (MIT), Cambridge, MA USA. ryuma@csail.mit.edu ics. Motion planning has been successfully applied to a number of dynamic legged robots [13][14]. In particular, we solve the motion planning problem by performing trajectory optimization using a direct collocation method[15][16]. A few studies have explored trajectory optimization for the dynamic task using robotic systems with actuator dynamics. Our observation is similar to that reported in [17]. Modelbased trajectory optimization based on the iterative linear quadratic Gaussian (iLQG) method was used in [17] for a pneumatic humanoid. On the other hand, we use blackbox identification of the pneumatic actuator and a different trajectory optimization algorithm and experiment with an underactuated control task; we believe that the results support the growing body of evidence that suggests that model-based design can partially overcome the perceived limitations of pneumatic actuators.

\section{Model-Based Optimal Control enhanced by SYSTEM IDENTIFICATION}

\section{A. Overview}

To realize motion planning for a robot system employing actuator dynamics and nonlinear constraints, we propose a combination of model-based trajectory optimization and system identification techniques.

Complex actuator dynamics are usually neglected because servo motors can act as nearly ideal torque sources in a rated operation. On the other hand, the dynamics of a fluid actuator used in the highly-dynamic legged robot cannot be ignored. Furthermore, it is essential to consider the surrounding phenomena associated with a real system for successful performance(Fig.1).

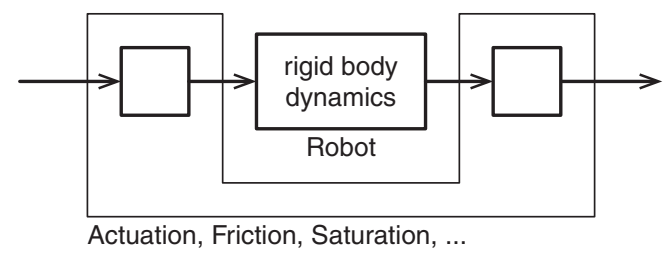

Fig. 1. Robot system surrounded by real-world constraints

Trajectory optimization with plain rigid body dynamics can produce good-looking but infeasible motions. To minimize the modeling error between numerical simulation and reality, we employ system identification techniques that use black-box and gray-box models. 


\section{B. Actuator Dynamics}

Detailed mathematical models of the pneumatic actuator have been proposed in previous studies. These analytical models use a combination of fluid dynamics equations and the states of valves and cylinder chambers. However, these models have a large number of related parameters that require appropriate identification but lack identifiability. Furthermore, a commercial pressure control valve is equipped with a built-in controller with a number of parameters which are impractical to model.

As an alternative approach, we identify the actuator dynamics using a black-box model that includes both fluid dynamics and valve dynamics. The black-box model can be applied not only to pneumatic actuators but also various types of actuators. In addition, the general structure of the blackbox model allows the use of general techniques for both parameter estimation and optimization. We can choose an appropriate model complexity by selecting the order of the model. The computational cost involved in the structure of the model is critical because it will impact the performance of our motion planning approach; it is desirable to achieve a fast evaluation of the system dynamics.

\section{Mechanical System and Friction model}

The equations of motion for the mechanical system can be described using the following general form of manipulator dynamics:

$$
H(q) \ddot{q}+C(q, \dot{q}) \dot{q}+G(q)=B(q) u
$$

where $q$ is the vector containing the joint positions/angles, $u$ the control input, $H$ the generalized mass matrix, $C$ the coefficient matrix of the Coriolis and centrifugal forces, and $G$ the gravity force.

There are various friction models that can be used for different conditions [18]. From our experimental analysis, we found that the pneumatic cylinder does not exhibit Stribeck effect (decreasing friction with increasing velocity). We use the Coulomb-viscous friction model with the tanh function, which is simple and numerically stable (eq.(2)).

$$
f=c_{1} \tanh \left(c_{2}\left(\dot{x}-c_{3}\right)\right)+c_{4} \dot{x}+c_{5}
$$

The friction model contains a bias term $c_{5}$ that represents an asymmetry property, which depends on the direction of the movement.

\section{Trajectory Optimization}

We consider motion planning for trajectory optimization to obtain an optimal trajectory satisfying the start configuration, the goal configuration, and all sets of constraints. We use a direct collocation method, which is implemented using the Matlab interface of the SNOPT solver. SNOPT [19] is employs the sparse sequential quadratic programming (SQP) method and is effective for solving nonlinear optimization problems. We provide analytical first derivatives (gradients) of the systems as input to the SNOPT solver.

\section{RoBOt Models}

\section{A. Pneumatic Actuator}

We use two types of systems which are driven by a pneumatic actuator. The actuator module shown in Fig. 2 is the basis of the systems.

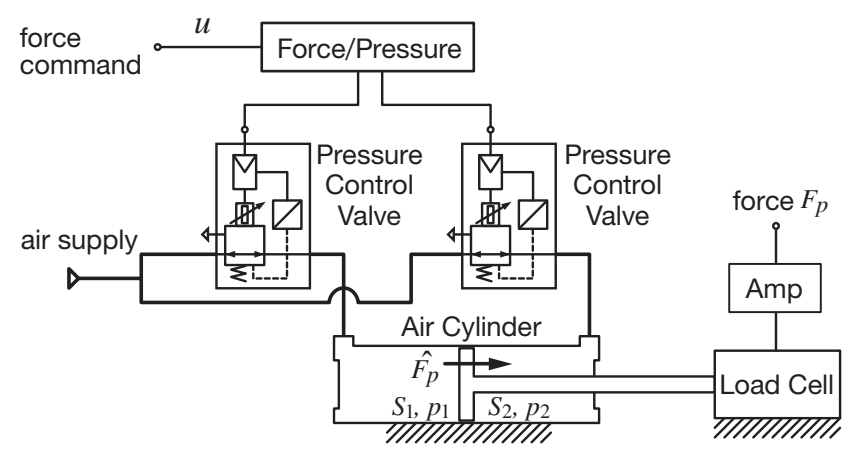

Fig. 2. Experimental setup of the pneumatic actuator

To minimize the nonlinear features of the input/output signal, we employ a differential pressure control method to generate a symmetric pressure command for each chamber of the air cylinder. On the basis of differential force generation, the desired pressure can be determined using the following equation.

$$
p_{1}=\left(F_{e q}+u / 2\right) / S_{1}, p_{2}=\left(F_{e q}-u / 2\right) / S_{2}
$$

where $S_{1}$ and $S_{2}$ represent the sectional area of the cylinder chambers and $p_{1}$ and $p_{2}$ the pressure of the cylinder chambers. The bias pressure $F_{e q}$ is given as the medial value of the supply pressure. The bias pressure allows continuous change of the pressure profile and avoidance of saturation caused by limited supply pressure. The $F_{e q}$ does not affect the compliance of the air cylinder because of the counterbalancing action of the double chambers.

\section{B. Pneumatic Cart-Pole}

A pneumatic cart-pole (Fig.3) exhibits nonlinear dynamics. We employ a decomposition approach for modeling the system to make it suitable for system identification. The system can be divided into a pneumatic part and a mechanical part. The subsystems are described with fluid dynamics and rigid-body dynamics respectively. Moreover, we add friction models to take into account the Coulomb force and viscous force, which are considered to perform force control in practice.

Because the cart-pole system (also known as inverted pendulum) is the basic control platform in the field of control theory, the cart-pole system model(eq.(4)) is mentioned in many previous studies.

$$
\begin{array}{r}
H(q)=\left[\begin{array}{cc}
m_{c}+m_{p} & m_{p} l \cos \theta \\
m_{p} l \cos \theta & m_{p} l^{2}
\end{array}\right], B=\left[\begin{array}{l}
1 \\
0
\end{array}\right] \\
C(q, \dot{q})=\left[\begin{array}{cc}
0 & -m_{p} l \dot{\theta} \sin \theta \\
0 & b_{p}
\end{array}\right] G(q)=\left[\begin{array}{c}
0 \\
m_{p} g l \sin \theta
\end{array}\right]
\end{array}
$$




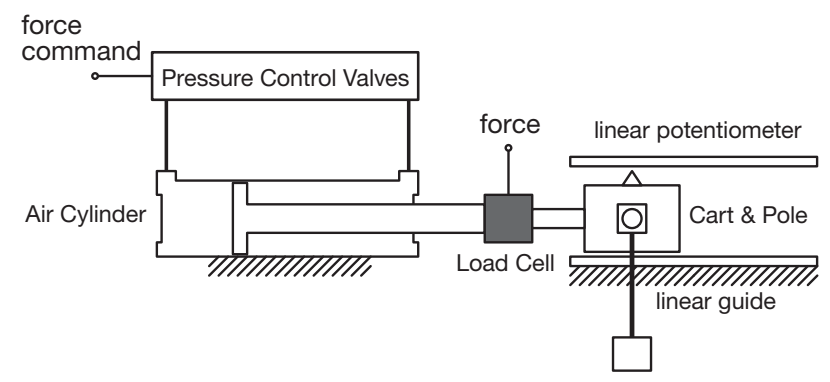

Fig. 3. Pneumatic cart-pole (inverted pendulum) system

The states represented by $q=[x, \theta]^{T}$ consists of the position of the cart and the angle of the pole. The parameters of our experimental system are as follows: $m_{c}=0.399 \mathrm{~kg}$, $m_{p}=0.1 \mathrm{~kg}, l=0.2 \mathrm{~m}, g=9.81 \mathrm{~m} / \mathrm{s}^{2}$, and $b_{p}=$ $0.0006 \mathrm{Nms} / \mathrm{rad}$.

\section{Compass Gait Walking Robot}

A compass gait walking robot is a simple legged robot with pneumatic toes (Fig.4). The robot is modeled as a hybrid system that involves discrete transitions of the dynamics. For the realistic simulation, each segment has its own mass and moment of inertia. The robot has a bisect body to reduce the number of actuators, which means that the angle of the body segment is determined as $\theta_{3}=\frac{\theta_{1}+\theta_{2}}{2}$.

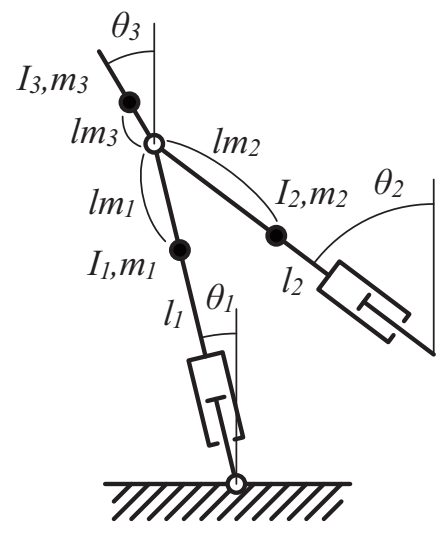

Fig. 4. Compass gait walking robot

\section{EXPERIMENTAL RESUlTS}

\section{A. System ID of the Pneumatic Actuator}

1) Fluid Dynamics: To capture the system dynamics, we use two kinds of excitation signal: chirp signals with various amplitudes, and random step sequences. During the measurement, the piston rod is fixed in the middle position. The range of the supply pressure is $0.0-0.5 \mathrm{MPa}$.

In black-box modeling, we investigate a polynomial model and a linear state space model, both of which are linear, timediscrete, and time invariant. The input $u$ is a force command, and the output $y$ is defined by eq.(5).

$$
y=\left[\hat{F}_{p}, F_{p}\right]^{T}, \hat{F}_{p}=S_{1} p_{1}-S_{2} p_{2}
$$

The force $F_{p}$ is measured by a load cell. The differential pressure force $\hat{F}_{p}$ is calculated from the pressure sensor measurements.

We use the autoregressive with exogenous terms (ARX) structure represented by eq.(6) as a polynomial model for the modeling of the pneumatic actuator.

$$
A(z) y(k)=B(z) u\left(k-n_{k}\right)+e(k)
$$

where $n_{k}$ is an input delay, $z$ is a delay operator, and $e(k)$ is the noise term. The ARX model has advantages, such as a simple model structure, efficient estimation of the parameters, capability to handle a multiple-input and multiple-output (MIMO) system, and the ability to model a noise term. The second-order ARX model (eq.(7)) is selected on the basis of an evaluation of the fitting error and computational cost. Fig.5 shows a comparison of the models. Although a higher-order state space model is comparable with the polynomial model, the result shows that the lower order ARX structure achieves higher fitness for modeling pneumatic dynamics.

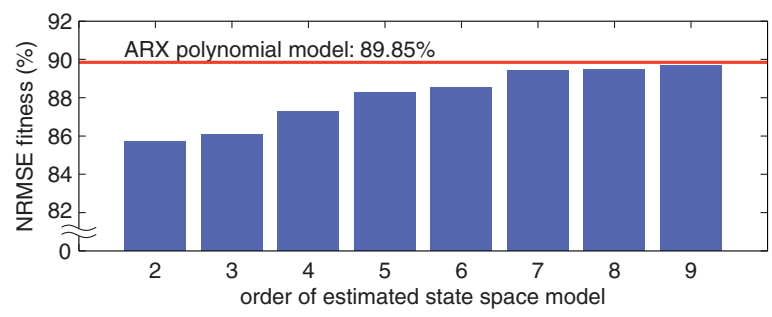

Fig. 5. Comparison of fitness for the ARX model and state space models

$$
\begin{aligned}
& A(z)=I+A_{1} z^{-1}, B(z)=B_{0}+B_{1} z^{-1} \\
& n_{k}=0
\end{aligned}
$$

The Matlab System Identification Toolbox is used for parameter estimation. eq.(8) shows the result of parameter estimation based on the least-squares method.

$$
\begin{array}{r}
A_{1}=\left[\begin{array}{cc}
-1.0144 & 0.0216 \\
-0.0527 & -0.9537
\end{array}\right] \\
B_{0}=\left[\begin{array}{l}
0.6619 \\
0.0764
\end{array}\right], B_{1}=\left[\begin{array}{l}
-0.6557 \\
-0.0847
\end{array}\right]
\end{array}
$$

Fig.6 and Fig.7 shows validation result of estimated pneumatic model. The obtained model fit is $89.85 \%$. The normalized root mean square (NRMSE) fit (\%) can be calculated using the following equation.

$$
f i t=100\left(1-\frac{\|y-\hat{y}\|}{\|y-\bar{y}\|}\right)
$$

where $y$ is the data, $\hat{y}$ is the model estimation, and $\bar{y}$ is the mean value of $y$. The results show that the model captures step response and frequency-dependent attenuation of the pneumatic dynamics. 

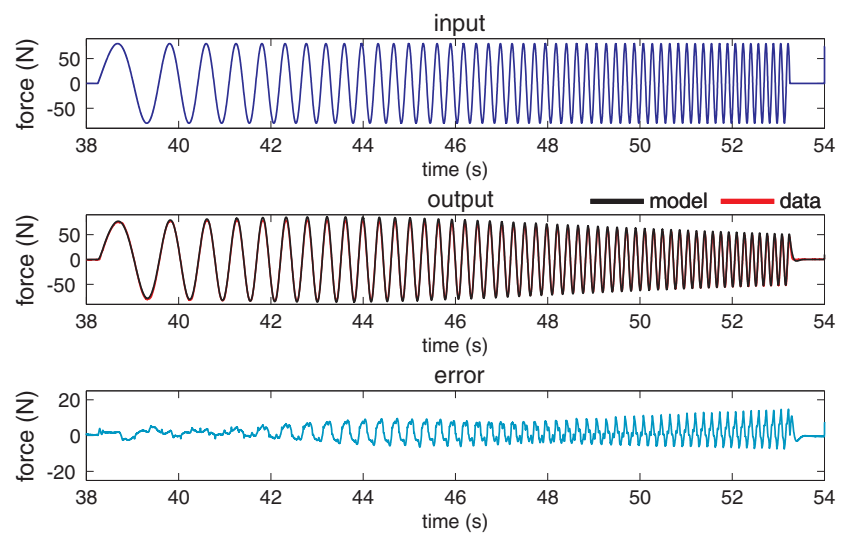

Fig. 6. Validation result using chirp signal.
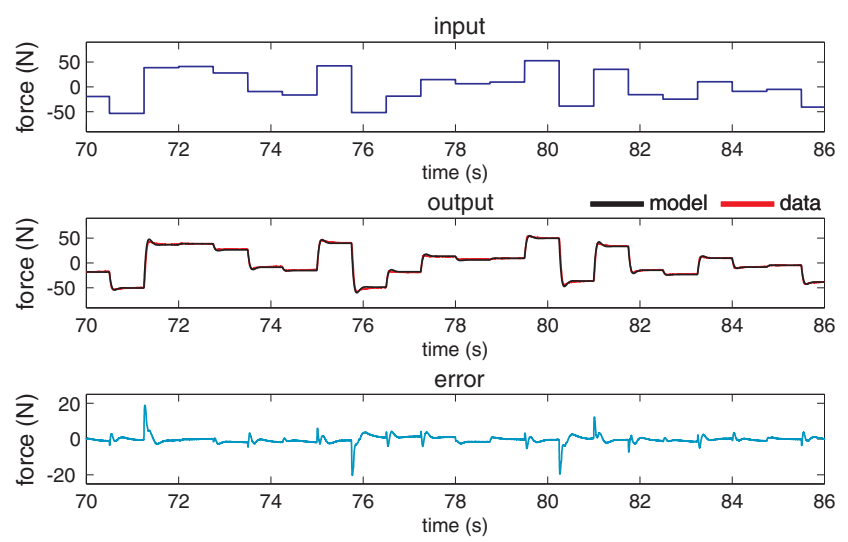

Fig. 7. Validation result using random stairs.

\section{B. Pneumatic Cart-Pole}

1) System Overview: We constructed an underactuated cart-pole system driven by the pneumatic actuator shown in Fig.8. In this system, the pneumatic system (eq.(8)), mechanical system (eq.(4)), and friction models (eq.(10) and eq.(11)) are connected in series. An air cylinder with a stroke of $300 \mathrm{~mm}$, and diameter of $\phi 20 \mathrm{~mm}$ is used for the system.

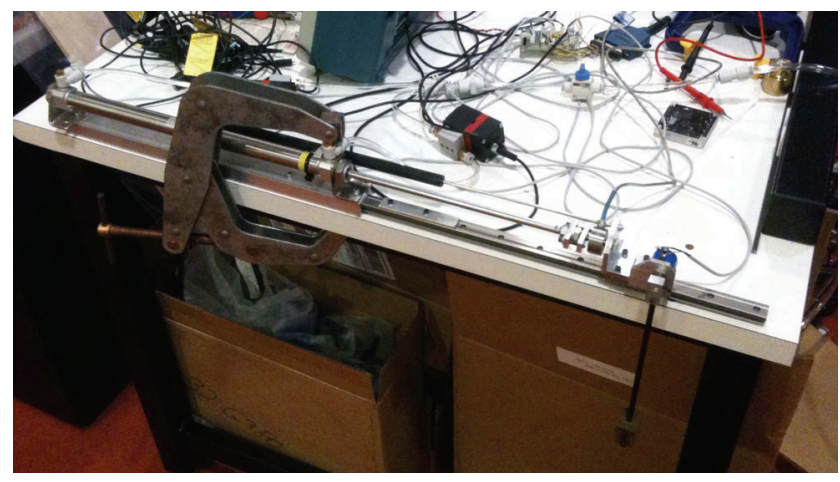

Fig. 8. Pneumatic cart-pole (inverted pendulum) experimental setup

2) Friction: Force data for system identification of the piston friction are measured by the load cell. eq.(10) shows the estimated parameters. The prediction error method is used to estimate the parameters of the gray-box friction model.

$$
F_{f p}=1.5145 \tanh (40.8926 \dot{x})+49.2734 \dot{x}
$$

The force data pertaining to the cart friction present in both linear potentiometer and linear guide are measured by the driving piston side of the load cell. The eq.(11) shows the estimated parameters.

$$
F_{f c}=5.7910 \tanh (63.7045(\dot{x}-0.0064))-2.3170
$$

Fig.9 shows the given data and obtained friction models. The result shows that the pneumatic cylinder has a considerable scale of frictional force, and the viscous force is dominant. The friction model for the cart has zero viscous friction and asymmetric Coulomb force.
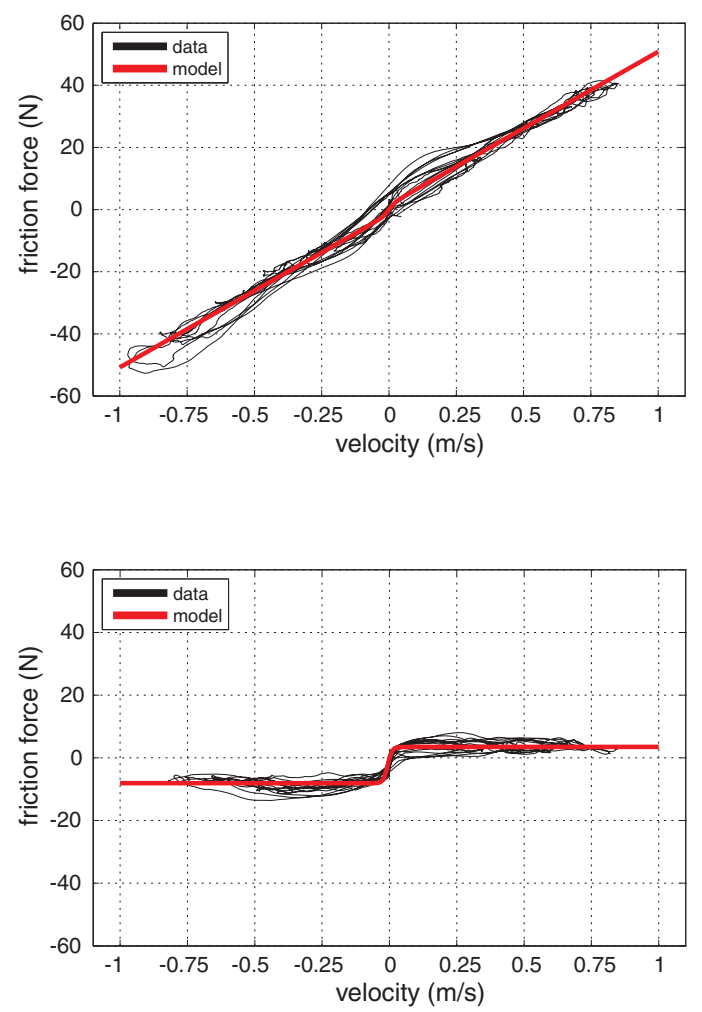

Fig. 9. Friction model for the piston (left) and for the cart (right)

3) Force Trajectory Control: A smooth trajectory was successfully derived from trajectory optimization. We used a swing-up task for the system. The nonlinear dynamics should be considered to achieve the task. The initial state and final state are set to $x_{0}=[0,0,0,0]^{T}$ and $x_{f}=[0, \pi, 0,0]^{T}$, where $x=[q, \dot{q}]^{T}=[x, \theta, \dot{x}, \dot{\theta}]^{T}$.

Fig.10 shows the comparison between the simulated position/angle and the measured position/angle. The results show that this method can perform realistic motion planning for the system using the pneumatic dynamics and friction models. The small variation of the trajectories in the ten trials indicates good repeat accuracy of the experiment. 

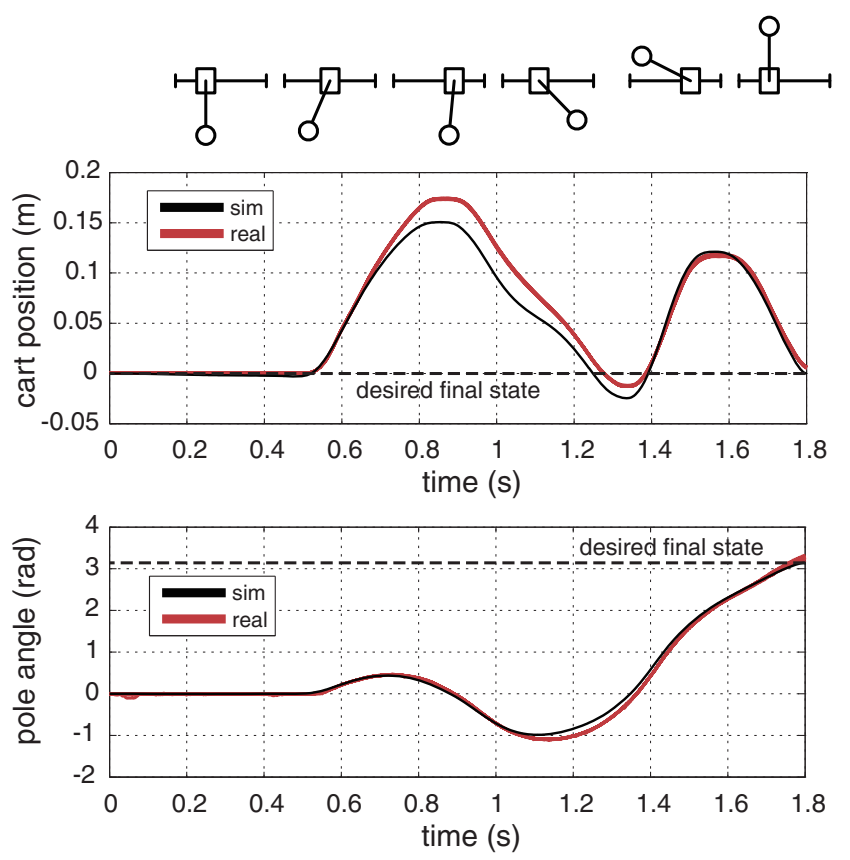

Fig. 10. Angle of the pole and position of the cart during swing-up with openloop force command. The overlapping red lines show 10 trials on real system.

Fig.11 shows the output force in both the simulation and the real experiment. The actual output force of the air cylinder exerted on the cart is reduced by the friction of the piston. The result shows that the proposed control method is able to produce a compensated force in practical situation. In the support phase of legged robots, force control is essential rather than the position/angle servo control.

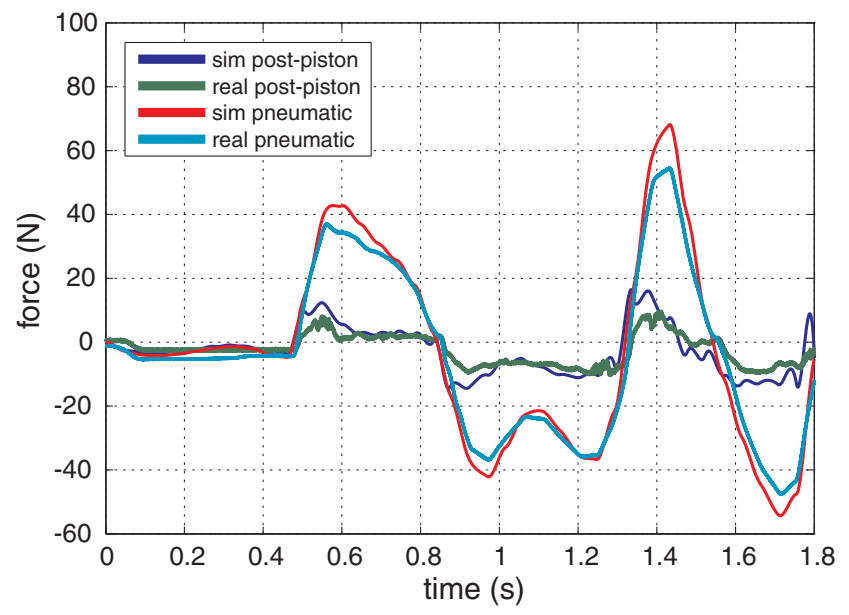

Fig. 11. The output force of the piston and the post-piston force reduced by frictions during swing-up

\section{Compass Gait Walking Robot}

1) System Overview: The waling robot is a planar walker that has three legs and four pneumatic toes. The hip joint is driven by an electric motor using harmonic gear. The robot consists of three components: two leg assemblies and a trunk with a bisection mechanism. The use of pneumatic actuators in the distal part of a legged robot can lead to low-inertia legs. The total mass of the robot is approximately $15 \mathrm{~kg}$. The leg length $1.05 \mathrm{~m}$ which is almost human-sized.

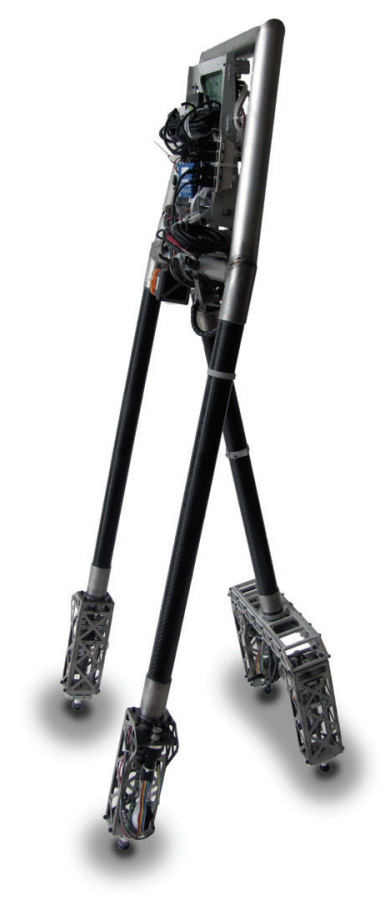

Fig. 12. A compass gait walking robot with pneumatic toes

2) Pneumatic Dynamics: eq.(12) shows the estimated parameters pertaining to the model of the pneumatic toes. The air cylinders used in the toes have a diameter of $\phi 40 \mathrm{~mm}$ and a stroke of $150 \mathrm{~mm}$.

$$
\begin{array}{r}
A_{1}=\left[\begin{array}{cc}
-1.0081 & 0.0240 \\
-0.0447 & -0.9502
\end{array}\right] \\
B_{0}=\left[\begin{array}{l}
0.6297 \\
0.0503
\end{array}\right], B_{1}=\left[\begin{array}{l}
-0.6141 \\
-0.0460
\end{array}\right]
\end{array}
$$

3) Trajectory Optimization: We perform trajectory optimization of half step walking for the compass gait walking robot (Fig.12). This robot is an underactuated system and is also a hybrid system, which includes collision dynamics. The initial state is $x_{0}=[0,0,1.045,1.0,0,0,0,0]^{T}$, and the final state is $x_{f}=[0,0,1.0,1.045,0,0,0,0]^{T}$, where $x=[q, \dot{q}]^{T}$, $q=\left[\theta_{1}, \theta_{2}, l_{1}, l_{2}\right]$. The desired step length is $0.25 \mathrm{~m}$.

To accelerate the searching of trajectories, we adopted a two-stage optimization approach for the walking robot. In the first stage, we perform trajectory optimization for the robot without pneumatic dynamics. The trajectory optimization considers constraints such as limitation of the actuator capacity and range of motion of the joint. Fig.13 is a stick figure representing the trajectory obtained.

In the second stage, we optimize the control input for the pneumatic actuator, which provides the required force for the walking motion. Fig.14 shows the optimized control input for 


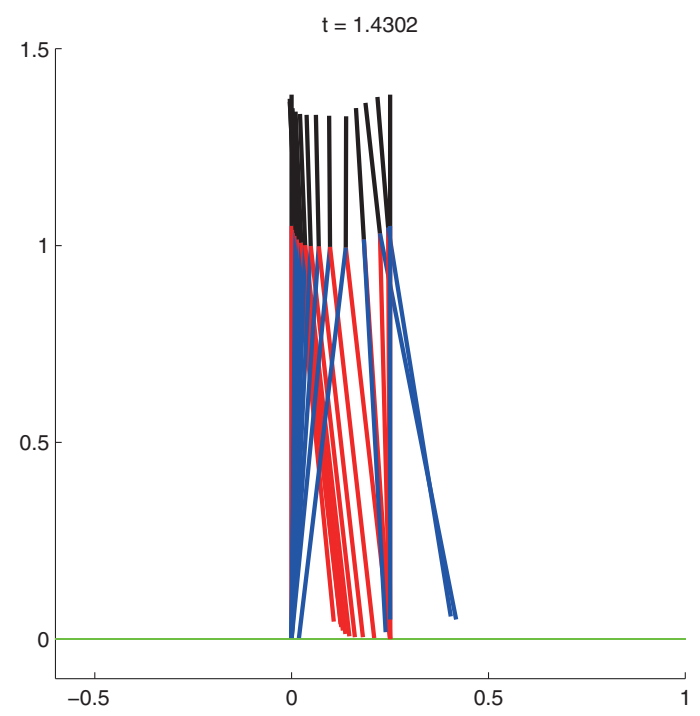

Fig. 13. Stick figure for an optimized trajectory of the half step walking

the pneumatic toes during the walking motion. The planned control input to the pneumatic system, which provides the desired output force, exhibits a complex profile. In contrast, a controller without the pneumatic model fails to produce required force output.

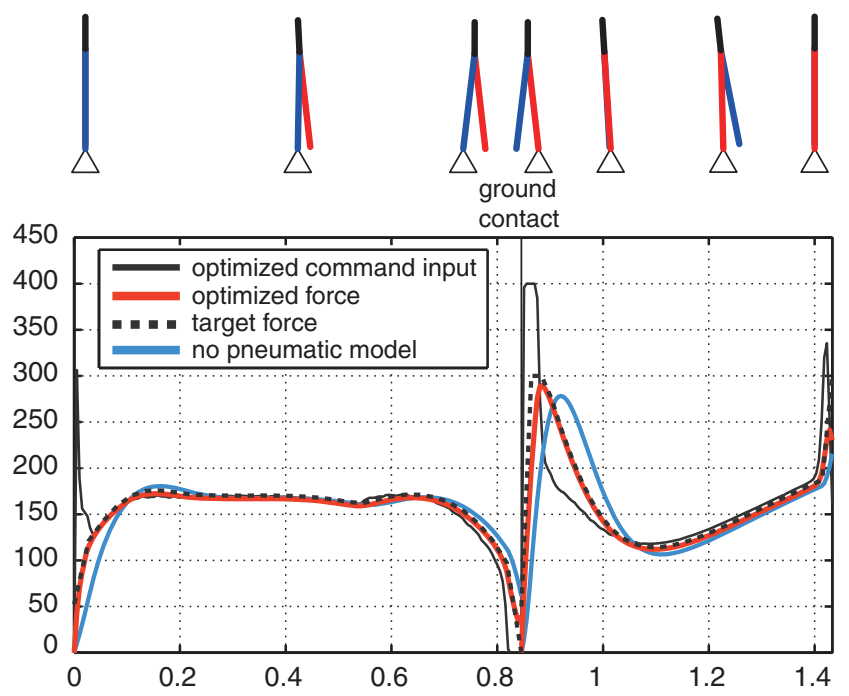

Fig. 14. Comparison between an optimized force output for the pneumatic actuator dynamics and a force output without pneumatic model for the half step walking

\section{CONCLUSION}

In this paper, we proposed a motion planning method for complex systems driven by pneumatic actuators. Method, which employs a model-based approach, was enhanced by the system identification technique. The obtained model of pneumatic dynamics realizes an $89.85 \%$ fit when compared to a measurement of real system. We demonstrated that planned trajectories are applicable to the real systems of pneumatic cart-pole and compass gait walking robot with pneumatic toes. The proposed method is potentially effective for the general motion planning of the robotic systems with highly-nonlinear dynamics. Our future study will focus on the development of a time-variant feedback controller for the obtained trajectory and verification of its effectiveness using a real robot.

\section{REFERENCES}

[1] Marc Raibert, Kevin Blankespoor, Gabriel Nelson, Rob Playter, and the BigDog Team. Bigdog the rough-terrain quadruped robot. In Proc. 17th World Congress of the International Federation of Automatic Control (IFAC), pages 10822-10825, Seoul, 2008.

[2] Sang-Ho Hyon. Compliant terrain adaptation for biped humanoids without measuring ground surface and contact forces. IEEE Transactions on Robotics, 25(1):171-178, 2009.

[3] Benjamin J. Stephens and Christopher G. Atkeson. Dynamic balance force control for compliant humanoid robots. In Proc. IEEE/RSJ Int. Conf. on Intelligent Robots and Systems (IROS 2010), pages 12481255, Taipei, 2010.

[4] Koh Hosoda, Takashi Takuma, Atsushi Nakamoto, and Shinji Hayashi. Biped robot design powered by antagonistic pneumatic actuators for multi-modal locomotion. Robotics and Autonomous Systems, 56(1):46-53, 2008.

[5] Björn Verrelst, Ronald Van Ham, Bram Vanderborght, Frank Daerden, Dirk Lefeber, and Jimmy Vermeulen. The pneumatic biped "Lucy" actuated with pleated pneumatic artificial muscles. Autonomous Robots, 18(2):201-213, 2005.

[6] Martjin Wisse and Richard Q. van der Linde. Delft Pneumatic Bipeds (Springer Tracts in Advanced Robotics). Springer, 2007.

[7] Ryuma Niiyama, Satoshi Nishikawa, and Yasuo Kuniyoshi. A biomechanical approach to open-loop bipedal running with a musculoskeletal athlete robot. Advanced Robotics, 26(3-4):383-398, 2012.

[8] Edmond Richer and Yildirim Hurmuzlu. A high performance pneumatic force actuator system: Part i-nonlinear mathematical model. Journal of Dynamic Systems, Measurement, and Control, 122(3):416425, 2000.

[9] Ming-Chang Shih and Shy-I Tseng. Identification and position control of a servo pneumatic cylinder. Control Engineering Practice, 3(9):1285-1290, 1995.

[10] J. E. Bobrow and B. W. McDonell. Modeling, identification, and control of a pneumatically actuated, force controllable robot. IEEE Transactions on Robotics and Automation, 14(5):732-742, 1998.

[11] D. Ben-Dov and S. E. Salcudean. A force-controlled pneumatic actuator for use in teleoperation masters. In Proc. Conf. IEEE Int Robotics and Automation, pages 938-943, 1993.

[12] Keith E. Gordon, Gregory S. Sawicki, and Daniel P. Ferris. Mechanical performance of artificial pneumatic muscles to power an ankle-foot orthosis. Journal of Biomechanics, 39(10):1832-1841, 2006.

[13] Alexander Shkolnik, Michael Levashov, Ian R. Manchester, and Russ Tedrake. Bounding on rough terrain with the littledog robot. Int. J. Rob. Res., 30(2):192-215, February 2011.

[14] M. Zucker, J.A. Bagnell, C.G. Atkeson, and J. Kuffner. An optimization approach to rough terrain locomotion. In Robotics and Automation (ICRA), 2010 IEEE International Conference on, pages 3589-3595, may 2010.

[15] C. R. Hargraves and S. W. Paris. Direct trajectory optimization using nonlinear programming and collocation. Journal of Guidance, Control, and Dynamics, 10(4):338-342, 1987.

[16] O. von Stryk and R. Bulirsch. Direct and indirect methods for trajectory optimization. Annals of Operations Research, 37:357-373, 1992.

[17] E. Todorov, Chunyan Hu, A. Simpkins, and J. Movellan. Identification and control of a pneumatic robot. In Proc. 3rd IEEE RAS and EMBS Int Biomedical Robotics and Biomechatronics (BioRob) Conf, pages 373-380, 2010.

[18] Soren Andersson, Anders Soderberg, and Stefan Bjorklund. Friction models for sliding dry, boundary and mixed lubricated contacts. Tribology International, 40(4):580-587, 2007.

[19] Philip E. Gill, Walter Murray, and Michael A. Saunders. Snopt: An sqp algorithm for large-scale constrained optimization. SIAM Journal on Optimization, 12(4):979-1006, 2002. 